\title{
USO DE ANTIBACTERIANOS EN INFECCIONES DEL TRACTO RESPIRATORIO INFERIOR, EN EL CENTRO MEDICO NAVAL, AÑO 2002.
}

\author{
JANNELLE C. MENDOZA L., CHRISTHY G. HOLGUíN C., JOSÉ R. JUÁREZ E. \\ DEPARTAMENTO ACADÉMICO DE FARMACOTECNIA Y ADMINISTRACIÓN FARMACEÚTICA - UNIVERSIDAD NACIONAL \\ MAYOR DE SAN MARCOS
}

\begin{abstract}
RESUMEN
El presente trabajo se desarrolló en el Centro Medico Naval «Cirujano Mayor Santiago Távara», considerando 206 historias clínicas de pacientes hospitalizados con tratamiento antibacteriano durante el periodo enero-diciembre/2002, en los Servicios de: Pediatría,Medicina Interna, Neumología y Geriatría; teniendo como objetivo evaluar la terapia antibacteriana en infecciones del tracto respiratorio inferior a fin de determinar la efectividad del uso de antibacterianos en los pacientes. De las historias analizadas; $33 \%$ correspondieron a exacerbación aguda de la bronquitis (E $\Lambda \mathrm{B}), 53 \%$ a neumonía adquirida en la comunidad (NAC) y $14 \%$ a exacerbación infecciosa de las bronquiectasias (EIB). La mayoría de los pacientes presentó síntomas comunes como tos $84 \%$, producción elevada de esputo $67 \%$ y fiebre $65 \%$. Los antibacterianos prescritos con mayor frecuencia fueron cefuroxima $36 \%$, ciprofloxacino $15 \%$ y claritromicina $15 \%$; prescritos como monoterapia en cefuroxima $44 \%$, claritromicina $17 \%$ y ceftriaxona $13 \%$; y como terapia asociada ceftriaxona/ciprofloxacino $8 \%$, cefuroxima/claritromicina $7 \%$, cefuroxima/ ciprofloxacino $7 \%$.
\end{abstract}

Se demostró que el uso de antibacterianos fue efectivo debido a que en el $73 \%$ de casos no hubo cambios en la terapia inicial; sin embargo, del $27 \%$ de los casos en que se hicieron cambios, no se encontró reporte de justificación en $58 \%$.

PALABRAS CLAVES: neumonía, neumonía adquirida en la comunidad, bronquitis, bronquiectasias, infecciones respiratorias, tratamiento antibacteriano.

\section{SUMMARY}

The present research was developed in the Centro Médico Naval "Cirujano Mayor Santiago Távara", considering 206 clinical historics of patients hospitalized with antibacterial treatment during the period January-December of 2002, in the Services of: Pediatrics, Internal Medicine, Pneumology and Geriatrics. Whose general objective was to evaluatc the antibacterial therapy in the infections of the inferior respiratory tract in order to determine the effectiveness of the antibacterial usage in patients.

Of the analyzed histories; $33 \%$ repayed to acute exacerbation of bronchitis (AEB), $53 \%$ to community-acquired pneumonia $(\mathrm{C} \wedge \mathrm{P})$ and $14 \%$ to infectious cxaccrbation of the bronchiectasias (IEB). most of the patients presented common symptoms as cough $84 \%$, high production of sputum $67 \%$ and fever $65 \%$.

The antibacterial drugs prescribed with more frequently were cefuroxime $36 \%$, ciprofloxacin $15 \%$ and claritromycin $15 \%$.

The antibacterial treatment prescribed for the patients was a monotherapy with cefuroxime $44 \%$, claritromycin $17 \%$ and ceftriaxon 13\%; and as a associatc therapy ceftriaxon/ciprofloxacin $8 \%$, cefuroxime/claritromycin $7 \%$, cefuroxime/ ciprofloxacin $7 \%$.

In conclusion, the antibacterial drugs used was effective because changes didn't exist ir the initial therapy. However, in the cases in which changes were made in the initial therapy $27 \%$, the justification was not reported in $58 \%$ of cases.

KEY WORDS: pneumonia, community- acquired pneumonia, bronchitis, bronchiectasias, respiratory infections, antibacterial treatment. 


\section{INTRODUCCIÓN}

Las infecciones de las vías respiratorias son una de las causas más importantes de morhimortalidad en muchos países; desarrollados y en vías de desarrollo; de ellas la neumonía adquirida en la comunidad (NAC), las exacerbaciones infecciosas de las bronquiectasias(EIB) y las exacerbaciones agudas de bronquitis $(E A B)$ tienen enorme relevancia dadas las consecuencias sociales y económicas que cau$\operatorname{san}^{[1,2,3,8]}$.

Las infecciones respiratorias adquiridas en la comunidad constituyen la causa más frecuente tanto de consulta como de prescripción de antibióticos, $40 \%$ en el tratamiento ambulatorio y $60 \%$ en el medio hospitalario ${ }^{\{2,4\}}$. En la mayoria de los casos el médico no dispone de resultados microbiológicos específicos en que basar la elección del tratamiento, estableciendo la decisión en forma empírica, en función de los microorganismos más probables que causan la infección y del conocimiento del patrón de sensibilidad a los antibacterianos en cada área geográfica ${ }^{\{1,9,12\}}$.

Nuestro estudio evaluó la prescripción antibacteriana en pacientes hospitalizados por infecciones respiratorias del tracto inferior para encontrar la proporción de prescripción antibacteriana usada tanto como monoterapia y como terapia asociada.

\section{MATERIAL Y MÉTODOS}

Tipo de estudio: Estudio de tipo observacional, descriptivo, retrospectivo y de corte longitudinal ${ }^{[5,6]}$.

Ubicación Temporal: Los datos corresponden a los meses enero a diciembre del año 2002.

Universo: Estuvo conformado por todos los pacientes con diagnóstico clínico de NAC, EIB y EAB; cuya infección fue adquirida ambulatoriamente y cumplian los siguientes criterios de inclusión y exclusión:

\section{a) Criterios de inclusión}

- Pacientes con diagnóstico definitivo de NAC, EAB, EIB; de ambos sexos y mayores de un año, que estuvieron hospitalizados durante el periodo de estudio en los Servicios de Medicina Interna, Neumología, Geriatría y Pediatría.

b)Criterios de exclusión.

- Pacientes con infecciones respiratorias de causas no bacterianas como: virus, hongos y por microorganismos específicos como: $M$. tuberculosis y Parini.
- Pacientes con insuficiencia renal crónica, insuficiencia hepática crónica; con radiografía o TAC de pulmones normales.

- Pacientes con diagnóstico de infección respiratoria intrahospitalaria (neumonía nosocomial).

\section{Muestra}

Confurmaron la muestra 206 pacientes, internados en algunó de los cuatro servicios motivo del estudio.

\section{Análisis de Datos Estadísticos}

Los datos obtenidos fueron procesados utilizando programas Microsoft Excel 2000 y SPSS versión 9.0 para Windows, permitiendo obtener los porcentajes, rangos, distribución, etc.

\section{Recolección de datos}

1. Se obtuvieron los datos de consumo diario de antibacterianos por los pacientes durante el período de estudio: cefepime, cefetamet, ceftazidima, ceftriaxona, cefuroxima, ciprofloxacino, moxifloxacino, azitromicina, claritromicina y ampicilina/sulbactam.

2. Se revisaron las historias clínicas de pacientes con tratamiento antibacteriano.

3. Los datos se registraron en una Ficha Farmacoterapéutica.

\section{RESULTADOS}

Tabla N¹: Número de pacientes con infecciones respiratorias según grupo etáreo.

\begin{tabular}{|l|c|c|}
\hline Edad & N & $\%$ \\
\hline$[1-20]$ & 50 & 24 \\
{$[20-39]$} & 42 & 20 \\
{$[39-58]$} & 33 & 16 \\
{$[58-77]$} & 37 & 18 \\
{$[77-96]$} & 44 & 21 \\
\hline TOTAL & 206 & 100 \\
\hline
\end{tabular}

$N=$ Número de prescripciones. 
El grupo etáreo con mayor número de infecciones respiratorias fueron los pacientes mayores de 48 años, representando hasta $40 \%$ de casos.

Tabla No2: Número de pacientes ingresados por trimestre

\begin{tabular}{|l|c|c|}
\hline Trimestres & N & $\%$ \\
\hline Enero - Marzo & 30 & 15 \\
Abril - Junio & 57 & 28 \\
Julio-Setiembre & 77 & 37 \\
Octubre - Diciem. & 42 & 20 \\
\hline TOTAL & 206 & 100 \\
\hline
\end{tabular}

$\mathrm{N}=$ Número de prescripciones.

El trimestre con mayor incidencia de infecciones respiratorias fue julio-setiembre, que coincide con los meses de invierno en la cual se registran las temperaturas mínimas.

Tabla № 3: Tiempo de hospitalización

\begin{tabular}{|l|c|c|}
\hline Dias & $\mathrm{N}$ & $\%$ \\
\hline$[1-7]$ & 59 & 29 \\
{$[8-14]$} & 92 & 45 \\
{$[15-21]$} & 29 & 14 \\
{$[22-+>$} & 26 & 13 \\
\hline TOTAL & 206 & 100 \\
\hline
\end{tabular}

$N=$ Número de prescripciones.

El tiempo de hospitalización en la mayoría de los casos fue de 8 a 14 días.
Tabla N2 4: Antibacterianos más prescritos durante el tratamiento.

\begin{tabular}{|l|c|c|}
\hline Antibacterianos & $\mathrm{N}$ & $\%$ \\
\hline Cefepime & 7 & 2 \\
Cefetamet & 34 & 10 \\
Ceftazidima & 9 & 3 \\
Ceftriaxona & 36 & 10 \\
Cefuroxima & 123 & 36 \\
Ciprofloxacino & 50 & 15 \\
Moxifloxacino & 16 & 5 \\
Azitromicina & 8 & 2 \\
Claritomicina & 50 & 15 \\
Amp/Sulbactan & 8 & 2 \\
\hline Total & 341 & 100 \\
\hline
\end{tabular}

$N=$ Número de prescripciones.

El antibacteriano más utilizado fue cefuroxima, seguida de claritromicina y ciprofloxacino.

Tabla Ne 5: Número de antibacterianos usados durante la terapia.

\begin{tabular}{|c|c|c|}
\hline Antibacterianos & N & $\%$ \\
\hline 1 & 110 & 53 \\
2 & 54 & 26 \\
3 & 29 & 14 \\
4 & 11 & 5 \\
5 & 2 & 1 \\
\hline Total & 206 & 100 \\
\hline
\end{tabular}

La monoterapia fue el tratamiento de elección en el $53 \%$ de casos. 
Tabla N²: Antibacterianos más usados como monoterapia y terapia asociada.

\begin{tabular}{|l|c|c|}
\hline Antibacterianos & $\mathrm{N}$ & $\%$ \\
\hline Cefuroxima & 123 & 44 \\
Claritromicina & 47 & 17 \\
Ceftriaxona & 36 & 13 \\
Cefetamet/Ciprofloxacino & 11 & 4 \\
Ceftriaxona/Ciprofloxacino & 22 & 8 \\
Cefuroxima/Claritromicina & 20 & 7 \\
Cefuroxima/Ciprofloxacino & 19 & 7 \\
\hline TOTAL & 154 & 100 \\
\hline
\end{tabular}

$N=$ Número de prescripciones.

La mayoría de casos tuvo como monoterapia cefuroxima, utilizado en infecciones leves y moderadas y como terapia asociada ceftriaxona/ciprofloxacino utilizado en infecciones severas.

Tabla $N^{2}$ 7: Duración del tratamiento antibacteriano

\begin{tabular}{|c|c|c|c|c|c|c|c|c|c|c|}
\hline$N^{\circ}$ de dias & \multicolumn{2}{|c|}{$[1-7]$} & \multicolumn{2}{|c|}{$[8-14]$} & \multicolumn{2}{|c|}{$[15-21]$} & \multicolumn{2}{|c|}{$[22-+]$} & \multicolumn{2}{|c|}{ Total } \\
\hline Antibacteriano & $\begin{array}{c}\text { № } \\
\text { Casos } \\
\end{array}$ & $\begin{array}{c}\% \\
\text { Casos } \\
\end{array}$ & $\begin{array}{c}N^{2} \\
\text { Casos } \\
\end{array}$ & $\begin{array}{c}\% \\
\text { Casos } \\
\end{array}$ & $\begin{array}{c}N^{\mathrm{o}} \\
\text { Casos }\end{array}$ & $\begin{array}{c}\% \\
\text { Casos }\end{array}$ & $\begin{array}{c}\text { № } \\
\text { Casos }\end{array}$ & $\begin{array}{c}\% \\
\text { Casos }\end{array}$ & $\begin{array}{c}\text { No } \\
\text { Casos }\end{array}$ & $\begin{array}{c}\% \\
\text { Casos }\end{array}$ \\
\hline Cefetamet & 0 & 0 & 18 & 153 & 16 & 47 & 0 & 0 & 34 & 100 \\
\hline Ceftazidima & 1 & 11 & 6 & 67 & 2 & 22 & 0 & 0 & 9 & 100 \\
\hline Ceftriaxona & 9 & 25 & 18 & 50 & 7 & 19 & 2 & 6 & 36 & 100 \\
\hline Cefuroxima & 10 & 8 & 24 & 20 & 69 & 56 & 20 & 16 & 123 & 100 \\
\hline Clindamicina & 0 & 0 & 6 & 40 & 5 & 33 & 4 & 27 & 15 & 100 \\
\hline Ciprofloxacino & 0 & 0 & 9 & 18 & 23 & 46 & 18 & 36 & 50 & 100 \\
\hline Moxifloxacino & 0 & 0 & 8 & 50 & 7 & 44 & 1 & 6 & 16 & 100 \\
\hline Azitromicina & 7 & 88 & 1 & 13 & 0 & 0 & 0 & 0 & 8 & 100 \\
\hline Claritromicina & 1 & 2 & 22 & 44 & 20 & 40 & 7 & 14 & 50 & 100 \\
\hline Amp/Sulb & 0 & 0 & 2 & 25 & 4 & 50 & 2 & 25 & 8 & 100 \\
\hline
\end{tabular}

La duración del tratamiento fue de 8 a 14 días para la mayoría de los antibacterianos: cefepime, cefetamet, ceftazidima, ceftriaxona y claritromicina. 
Tabla № 8: Cambio en la terapia antibacteriana

\begin{tabular}{|c|c|c|}
\hline Cambio & N & $\%$ \\
\hline NO & 150 & 73 \\
SI & 56 & 27 \\
\hline Total & 206 & 100 \\
\hline
\end{tabular}

$N=$ Número de preseripciones.

Se observaron cambios en la terapia antibacteriana inicial en el $27 \%$ de casos.

Tabla № 9: Motivo del cambio en la terapia antibacteriana

\begin{tabular}{|l|r|r|}
\hline Motivo & $\mathrm{N}$ & $\%$ \\
\hline Agravación del cuadro & 8 & 4 \\
Justificación no reportada & 119 & 58 \\
Persistencia de fiebre & 62 & 30 \\
Persistencia de otros síntomas & 12 & 6 \\
Otros & 5 & 2 \\
\hline TOTAL & 206 & 100 \\
\hline
\end{tabular}

$N=$ Número de prescripciones.

La justificación del camoio de terapia no se registra en la mayoría de casos.

\section{DISCUSIÓN}

Se encontró que las infecciones respiratorias ocurrieron en pacientes del sexo masculino representando el $69 \%$, a diferencia de estudios previos que no encontraron diferencia significativa ${ }^{[6,22,14]}(44 \%$ y $56 \%$ respectivamente), esta se explica porque la población, por tratarse de una institución militar, es en su mayoría masculina.

La edad de los pacientes varió entre 1 a 96 años siendo el grupo etáreo de mayor incidencia de 58 a 96 años. Según estudios anteriores, en el Perú las infecciones del tracto respiratorio inferior son consideradas como causa importante de mortalidad en niños menores de 1 año; y morbimortalidad en niños de 1 a 4 años y en personas mayores de 60 años $\{3,7,20\}$,
De los 206 pacientes, 109 (53\%) fueron diagnosticados con NAC, 68 (33\%) fueron diagnosticados con EAB y $29(14 \%)$ con EIB. La incidencia de las infecciones respiratorias, considerando períodos trimestrales fue mayor en el trimestre julio - septiembre con $37 \%$; probablemente debido al aumento de humedad relativa en el ambiente, asociada a la disminución de temperatura, que alcanza sus máximos niveles en esta época del año ${ }^{[y]}$.

Durante el tratamiento, la frecuencia de uso de antibacterianos fue $36 \%$ para cefuroxima, $15 \%$ para claritromicina y $15 \%$ para ciprofloxacino. Estos antibacterianos están incluidos en esquemas terapéuticos de tratamiento hospitalario de infecciones respiratorias adquiricas en la comunidad, los mismos que incluyen cefalosporinas de segunda (cefuroxima) y tercera generación (ceftriaxona, cefotaxima), macrólidos (azitromicina, claritromicina), fluoroquinolonas de segunda (ciprofloxacino), tercera (levofloxacino) y de cuarta generación (trovafloxacino y moxifloxacino) ${ }^{[0,11,23\}}$.

En el $73 \%$ de los casos no hubo cambios en la terapia antibacteriana, lo que indica un acierto en el manejo del paciente, coincidiendo con valores encontrados en otros estudios realizados ${ }^{11}$. De estos, en el $58 \%$ de los casos no se reportó una justificación por el facultativo siendo necesario conocer el motivo del cambio de terapia inicial, mientras que la persistencia de fiebre representó la justificación en $30 \%$ de los casos.

\section{CONCLUSIONES}

1. Se cvidencia que el tratamiento inicial en infecciones del tracto respiratorio inferior es efectivo en el $73 \%$ de casos.

2. Se observa que el tratamiento para infecciones del tracto respiratorio inferior cumple con los estándares internacionales de tratamiento.

3. La prescripción más frecuente en infecciones del tracto respiratorio inferior fue la cefuroxima como monoterapia $44 \%$ de casos.

\section{REFERENCIAS BIBLIOGRÁFICAS}

1. Llanos F. Mayca J. y Contreras C. 2002. Características de la prescripción de antibióticos en los consultorios de medicina interna del hos" pital Cayetano Heredia de Lima - Perú, Revista española de Salud Pública 2002; N"76, pp. 207. 214, mayo-junio, Lima. 
2. Organización Mundial de la Salud. 2001. Protocolo: Estudio sobre la prescripción, el uso y las Reacciones Adversas a los antimicrobianos en pacientes hospitalizados. Oficina General de Epidemiologia (OGE-RENACE), Ministerio de Salud, Lima.

3. Ramirez J., Blondeau J., Schentag J., Soto J. 2001. Parámetros innovadores para evaluar las opciones terapéuticas en las Neumonías, $X$ Congreso de la Asociación Panamericana de Infectología, junio 2001, Guadalajara.

4. Morsey I., Hunter P. 2000 . The importance of the ability of an antimicrobial agent to Hill the pathogen, Maxim Medical, vol. 3, pp. $33-48$, Morelos.

5. Miravitlles M., Ros F, Cobos. A. 2001. Interna* cional Journal of Clinical Practice, Medicina Clinica., Barcelona; 55 (7): pp. 437-441.

6. Luna J. 2000. El papel del moxifloxacino en las neumonias, Evaluación Clinica del moxifloxacino en el tratamiento de infecciones respiratorias, Guadalajara.

7. Calmaggi A. 1999. Guías para el diagnóstico y tratamiento de la neumonia adquirida en la comuni- dad en adultos, Sociedad Argentina de Infectología, Buenos Aires, Diciembre.

8. Murray y Nadel. 2000. Texbook of Respiratory Medicine, tercera edición, pp. 919-932, Saunders Company, Barcelona.

9. García J., Barberan J., Campanero M., Dorca J., Zalacin R. 2001. Actualización en el tratamiento de las infecciones respiratorias; Medicina Clínica, Barcelona , 2 (2).

10. Censa J., Gatell J. Jiménez T. y Prąst G. 2000, Guía de Terapéutica Antimicrobiana, décima edición, Barcelona, Editorial Masson S.A..

11. Le Grand A., Hogerzeil H., Haaijer F. 1999. Intervention research in racional use of drugs: A review health policy and planning, Methodist Health Care System, vol. 14, pp. 89-102.

12. Martínez M, Gómez $\mathrm{C}$ y Cabanillas M. 1997. Patogénesis, diagnosis and treatment of bronchiectasias, Bronquiectasias Medicine, 7 (38): pp. 1653-1657.

13. García J, Trujillano I., Fresnadillo M., Iglesias E. 1999. Moxifloxacino en la Práctica, Revista Bayer, 2: pp. 45-58. 